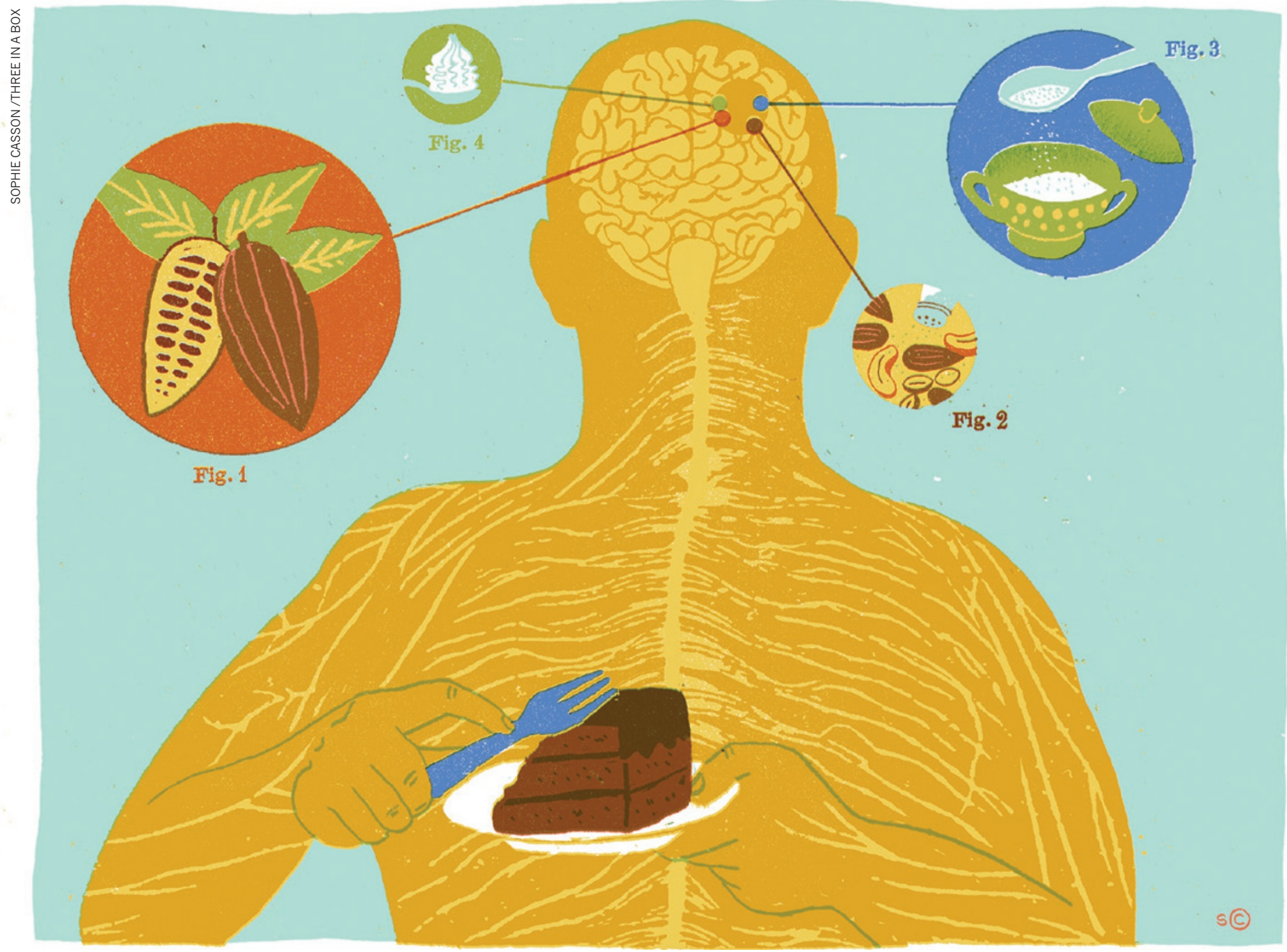

NEUROSCIENCE

\title{
Hardwired for taste
}

\section{Research into human taste receptors extends beyond the tongue to some unexpected places.}

\section{BY BIJAL P. TRIVEDI}

A mouthful of bittersweet chocolate cake with a molten centre can trigger potent memories of pleasure, lust and even love. But all it takes is one bad oyster to make you steer clear of this mollusc for life. Neuroscientists who study taste are just beginning to understand how and why the interaction of a few molecules on your tongue can trigger innate behaviours or intense memories.

The sensors in our mouths that detect basic tastes - sweet, salty, bitter, sour and umami, and arguably a few others - are only the start of the story (see 'The finer points of taste, page S2). The way the brain represents these tastes is just as important. Researchers have recently developed a 'gustotopic map' based on the idea that, just as each taste bud on the tongue responds to a single taste, so there are regions of the brain that are similarly dedicated ${ }^{1}$.

The other recent revelation in taste research is that the receptors that detect bitter, sweet and umami are not restricted to the tongue. They are distributed throughout the stomach, intestine and pancreas, where they aid the digestive process by influencing appetite and regulating insulin production. They have also been found in the airways, where they have an impact on respiration, and even on sperm, where they affect maturation. A better understanding of what they do and how they work could have implications for treating conditions ranging from diabetes to infertility.

\section{BRAIN MAP}

After discovering the sensors for the five basic tastes, Charles Zuker, a Howard Hughes Medical Investigator now at Columbia University,
New York, and geneticist Nicholas Ryba, of the National Institute of Dental and Craniofacial Research in Bethesda, Maryland, embarked on a logical follow-on project. Their goal, Zuker says, was to determine how the brain "transforms detection into perception".

Results of previous studies into taste representation in the brain "have been confusing", says Ryba. One of the leading theories was that the gustatory cortex - the primary brain region responsible for taste perception - was 'broadly tuned', with each neuron responding particularly well to one taste but still able to respond to others. Moreover, the neurons were thought to be distributed evenly. This model seemed to be consistent with smell, the other chemical sense, go.nature.com/bw66nx 


\section{THE SECRET LIVES OF TASTE RECEPTORS}

\section{The ability to sense chemicals has whole-body applications}

Taste receptors promote survival by detecting nutritious foods and helping us avoid toxic ones. But these receptors exist beyond the tongue and digestive system. How do they contribute to the survival of the species?

In a bid to find out, Thomas Finger, a neurobiologist and co-director of the University of Colorado's Rocky Mountain Taste and Smell Center in Aurora, has been studying solitary chemoreceptor cells (SCCs). In 2003, he discovered SCCs in the nasal cavities of rats and mice; more recently, he and others have found SCCs expressing taste receptors in the human nose. The rodent SCCs contain T2Rs (bitter receptors) and all the rest of the cellular machinery found in bitter taste cells on the tongue. In 2010, Finger and colleagues showed ${ }^{6}$ that bitter compounds that tickle these receptors trigger apnoea: "they stop breathing, they cough and sneeze," he says. The receptors, which are exposed to harmful irritants in the air as well as to compounds produced by bacteria growing in the nasal cavity, transmit their signal to the trigeminal nerve, which temporarily inhibits breathing. This response, he hypothesizes, is to stop the irritant from being inhaled deep into the lungs.

It's not only the T2Rs that are found in hard-to-explain places. The T1Rs, responsible for sensing sweet and umami, exist in the airways too, although what they do there remains unknown. "It is easier to understand the T2Rs than the T1Rs," says Finger, "T2Rs always seem to be present in the context of detecting toxins. The T1Rs seem to have other functions here that are unclear."

Collections of bitter receptors are also found on tiny finger-like projections called cilia on human airway epithelium cells. When the researchers stimulated the receptors using bitter compounds, such as nicotine or quinine, the cilia waved back and forth vigorously, helping to clear the airways of irritating compounds.

Researchers at the University of Maryland School of Medicine in Baltimore discovered T2Rs on smooth muscle cells in the human airway ${ }^{7}$. Exposure to bitter compounds caused these cells to relax. Testing this effect in asthmatic mice, where smooth-muscle contraction narrows the airways and obstructs breathing, had the same effect: bitter compounds relaxed the smooth muscle and improved breathing.

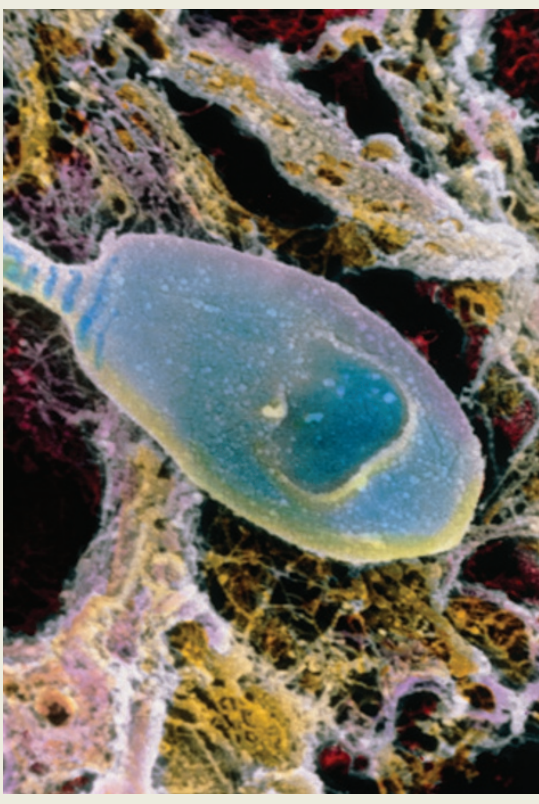

A sperm trying to fertilize an egg. Umami receptors, found on the sperm's surface, are thought to help control the release of DNA.

There are many more locations where the presence of taste receptors is enigmatic. A team of researchers led by Ingrid Boekhoff of the Walther Straub Institut for Pharmacology and Toxicology in Munich, Germany, recently found ${ }^{8}$ that mouse and human spermatozoa express T1R1 and T1R3, the umami receptor. Murine sperm that lack T1R1 had an increased rate of spontaneous acrosome reaction - a disgorging of DNA that normally only happens when the sperm meets an egg. The mutant sperm also had higher levels of calcium and a second messenger molecule called cAMP. Boekhoff and her colleagues suggest that the umami receptor somehow keeps the sperm in a quiet state by controlling calcium ions and cAMP - which are known to influence the acrosome reaction - until it reaches an egg.

The bitter receptor T2R 5 is also found in the testes ${ }^{9}$; deleting these cells leads to smaller testes and a huge reduction in the number of mature sperm. According to Robert Margolskee, associate director of the Monell Chemical Senses Center in Philadelphia, Pennsylvania, it is not clear what the bitter or umami receptors are doing in these locations. "But we have the tools to start figuring it out," he says. "Check back in five years and we'll have a pretty good idea." - B.T. which produces no recognizable patterns in the brain. Odour recognition is a matter of combinatorial processing: small differences in firing patterns across large populations of neurons represent a characteristic smell.

The research that led to these conclusions suffered from poor spatial resolution, however. To address this shortcoming, Zuker and Ryba's team used functional brain-imaging techniques to investigate how individual taste qualities stimulate neural activity in the gustatory cortex on a fine scale. Their tools included two-photon calcium imaging, which reveals processes deeper inside the brain, and with greater spatial resolution, than conventional single-photon methods. They found that dropping any of several bitter liq uids onto the tongue of an animal that had been anaesthetized (both for convenience and to replicate conditions in previous studies) consistently activated the same small group of neurons in the gustatory cortex. These neurons did not light up in response to sweet, savoury or salty liquids.

The team ran the same experiment for the other tastes and found that sweet, salty and umami each had its own distinct cluster, or hotspot, of neurons. The only hotspot they couldn't locate was for sour tastes.

These findings contradict previous ideas about how the brain processes taste. Seeing the first hotspot was "stunning", recalls Ryba. "On the one hand it was very surprising: at the level of the tongue, the sweet and bitter cells and receptors are intermingled, yet at the level of the cortex they are separated by 2.5 millimetres" - a surprisingly large distance that could span hundreds of neurons. On the other hand, Ryba can see the logic of this organization: separating the bitter hotspot from the sweet, for example, means that bitterness can be wired to a brain area that drives aversion, whereas sweetness can be wired to attraction.

So far the gustatory map is sparse, with just four identified hotspots. But other areas nearby might also be used for taste coding, possibly involving other senses, speculates Ryba. These areas might represent mixtures of tastes or perception of flavour.

Finding these hotspots is "a breakthrough" that provides a "basic underlying principle of how the cortex is organized", says Susan Travers, a neuroscientist at Ohio State University in Columbus. Nevertheless, Travers thinks the new map is oversimplified. Previous experiments found that a neuron "doesn't respond to just one stimulus", she says. For example, "you would expect the sweet hotspot to have some smaller responses to other stimuli", such as salt or umami.

Sidney Simon, a neuroscientist at Duke University in Durham, North Carolina, who specializes in gustatory physiology, says that Ryba and Zuker's experiments are "technically spectacular" - but he also has concerns. He says it is strange that they didn't find a sour hotspot, and recommends that they explore whether it lies in a different area or whether sour detection is dispersed throughout the brain. He adds that these 
experiments should be performed on conscious animals, which, in terms of smell at least, respond differently from anaesthetized ones.

Zuker takes the criticisms in his stride. He's not perturbed by the lack of a sour hotspot and explains that, unlike the other four tastes, sour or acid detection - is also involved with pain. "When you put a drop of acid on your finger it burns, you aren't getting a sour lemony taste," he says. So the cortical representation is likely to be more complicated than a single hotspot. Other tastes don't have this type of conflict, he adds.

As for Travers' comment that the hotspots appear to be too specialized, Zuker concedes that there may be some sensory crossover, with some cells in the bitter hotspot, for example, also responding to other tastes. The key, he stresses, is that there is overall topographic segregation, and that the vast majority of neurons within a hotspot are selective for just one taste.

Zuker and Ryba now plan to explore how taste mixtures are encoded, and then how sweet and bitter tastes, after being detected by their corresponding cortical fields, can trigger such exquisite behaviours - from attraction to aversion, from pleasure to rejection.

\section{OUT OF THE MOUTH}

While Zuker and Ryba use receptors to explore how taste relates to emotion, memory and learning, other researchers hope to explain what taste receptors are doing in other parts of the body.

If taste receptors seem out of place anywhere other than the mouth, this is only because they were first found in taste buds on the tongue, says neurobiologist Thomas Finger, co-director of the University of Colorado's Rocky Mountain Taste and Smell Center in Aurora. Taste buds are simply a way of sensing chemicals, so they can have functions unrelated to detecting the flavour of food. And they are surprisingly common in the body (see 'The secret lives of taste receptors'), although their presence is sometimes baffling. "We don't know the function of these receptors in more places than we do know them," says Finger.

It is not surprising that some of the betterunderstood examples are in the digestive system. The T1R2/T1R3 sweet receptor is found on $\mathrm{K}$ - and L-type enteroendocrine cells in the intestine. These cells secrete hormones called incretins, which in turn stimulate insulin production. The sweet receptors neatly explain a phenomenon that had mystified physiologists for more than 50 years: that eating glucose triggers significantly more insulin than injecting it directly into the bloodstream. Neuroscientist Robert Margolskee, now associate director of the Monell Chemical Senses Center in Philadelphia, Pennsylvania, realized that if there were receptors in the intestine
TASTE CIRCUITS

with taste receptors are found throughout the body (shown in green) ${ }^{10}$ 列 cts - that carry only secretions produced by the body - their purpose is
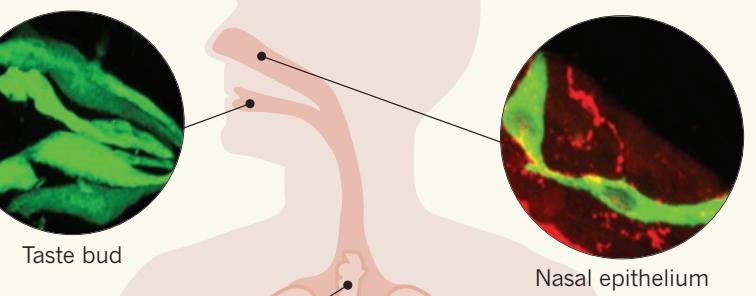
Nasal epithelium
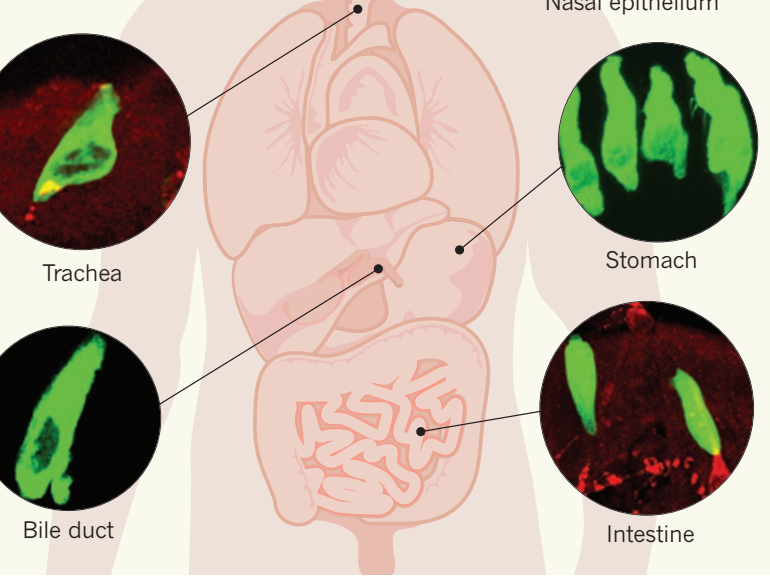

that could detect glucose and trigger the release of hormones, this would provide the missing link for the so-called incretin effect. In 2007, his hypothesis proved correct as his team helped to find the sweet receptor on L cells in the human duodenum $^{2}$ and showed that these cells produce the gastrointestinal incretin hormone GLP-1, which stimulates insulin production and sends a satiety signal to the brain. Blocking or deleting these sweet receptors decreases insulin release.

Margolskee worked with a group from the University of Liverpool, UK, to show in mice that when sweet receptors detect glucose, cells adjacent to the L cells make a glucose transporter that draws sugar in from the intestine ${ }^{3}$. Artificial sweeteners also trigger the glucose transporter and lead to a spike in insulin - this is a concern, but is unlikely to cause clinical hypoglycaemia (low blood glucose), Margolskee says.

"Sweet receptors, traditionally associated with just the mouth, were in the gut and essentially 'tasting' the sugar a second time," says Anthony Sclafani, a behavioural neuroscientist at the City University of New York. This 'second tasting triggers glucose transport into the cells and bloodstream, and the faster this happens, the more insulin will be released. "It's an incredibly important finding for the control of blood sugar," he says, adding that it was surprising that artificial sweeteners, which were thought to influence only the tongue, also trigger changes in the gut.

Other T1R receptors in the digestive system also play a role in appetite and blood sugar control. In the stomach, cells carrying the T1R3 receptor, which aids detection of both sugar and amino acids, secrete the hunger hormone ghrelin when they encounter carbohydrates and protein, encouraging eating when important nutrients are available.

For bitter tastes, however, the T2R receptors in the digestive system seem to have contradictory functions. In 2011, Belgian researchers showed that bitter-tasting compounds that reach the stomach of mice initially trigger the release of ghrelin, stimulating eating as usual ${ }^{4}$. But after 30 minutes, food intake decreased, as did gastric emptying, keeping the food in the stomach. This curbs the appetite by prolonging the sense of fullness and satiety - perhaps to prevent the ingestion of toxic food.

"It's too early to know whether this is a normal satiety response or this is mimicking a response to a toxin," says Roger Cone, a biophysicist at Vanderbilt University in Nashville, Tennessee. It seems counter-intuitive that eating a bitter compound, which is thought to signal a toxin, actually stimulates the appetite. Yet this is an effect that has been known about for centuries. Indeed, the Romans drank wine infused with bitter herbs to prime the appetite and prevent overeating. And, the authors speculate, stimulating bitter receptors in the gut could potentially be used to treat certain eating disorders.

Bitter receptors are also present in the large intestine. In 2009, researchers at the University of Shizuoka in Japan showed that activation of T2Rs in the colon of humans and rats stimulates the secretion of anions, inducing water to enter and causing diarrhoea ${ }^{5}$. The authors suggest that this acts as a defence against dangerous organisms and irritants.

Investigating the function of the taste receptors distributed throughout the body could help clinicians tackle diseases ranging from eating disorders to diabetes. Digesting all this information will take time, but it is clear that the function of taste receptors goes far beyond the pleasure of eating chocolate cake.

Bijal P. Trivedi is a freelance science writer based in Washington DC.

1. Chen, X. et al. Science 333, 1262-1266 (2011)

2. Jang, H. J. et al. Proc. Natl Acad. Sci. USA 104 15069-15074 (2007)

3. Margolskee, R. F. et al. Proc. Natl Acad. Sci. USA 104 15075-15080 (2007).

4. Janssen, S. et al. Proc. Natl Acad. Sci. USA 108, 2094-2099 (2011).

5. Kaji, I. et al. Am. J. Physiol. Gastrointest. Liver Physiol. 296, G971-G981 (2009).

6. Tizzano, M. et al. Proc. Natl Acad. Sci. USA 107 3210-3215 (2010).

7. Deshpande, D. A. et al. Nature Med. 16, 1299-1304 (2010).

8. Meyer, D. et al. PLoS One 7, e32354 (2012).

9. Li, F. \& Zhou, M. Mol. Hum. Reprod. 18, 289-297 (2012)

10.Finger, T. E. \& Kinnamon, S. C. Biol. Rep. 3, 20 (2011). 